\title{
Retention and extinction of delay eyeblink conditioning are modulated by central cannabinoids
}

\author{
Adam B. Steinmetz and John H. Freeman ${ }^{1}$ \\ Department of Psychology, The University of lowa, lowa City, lowa 52242, USA
}

\begin{abstract}
Rats administered the cannabinoid agonist WIN55,212-2 or the antagonist SR141716A exhibit marked deficits during acquisition of delay eyeblink conditioning, as noted by Steinmetz and Freeman in an earlier study. However, the effects of these drugs on retention and extinction of eyeblink conditioning have not been assessed. The present study examined the effects of WIN55,212-2 and SR141716A on retention and extinction of delay eyeblink conditioning in rats. Rats were given acquisition training for five daily sessions followed by one session of retention training with subcutaneous administration of $3 \mathrm{mg} / \mathrm{kg}$ of WIN55,212-2 or $5 \mathrm{mg} / \mathrm{kg}$ of SR141716A and an additional session with the vehicle. Two sessions of extinction training were then given with WIN55,212-2, SR141716A, or vehicle. Retention and extinction were impaired by WIN55,212-2, whereas SR141716A produced no deficits. The extinction deficit in rats given WIN55,212-2 was observed only during the first session, suggesting a specific impairment in short-term plasticity mechanisms. The current results and previous findings indicate that the cannabinoid system modulates cerebellar contributions to acquisition, retention, and extinction of eyeblink conditioning.
\end{abstract}

The mammalian brain contains a heterogeneous distribution of cannabinoid-1 receptors (CB1R) with high levels in the substantia nigra, hippocampus, cerebral cortex, and cerebellum (Herkenham et al. 1990, 1991). The cerebellum contains particularly high levels of CB1Rs in the molecular layer of the cerebellar cortex (Herkenham et al. 1990, 1991; Ong and Mackie 1999). Previous studies have examined CB1R function during acquisition of delay eyeblink conditioning (dEBC), which depends on synaptic plasticity mechanisms within the cerebellar cortex (Lavond and Steinmetz 1989; Chen et al. 1996, 1999; Garcia et al. 1999; Attwell et al. 2001; Nolan and Freeman 2006; Thompson and Steinmetz 2009). Rats administered the CB1R agonist WIN55,212-2 or the antagonist SR141716A during acquisition of dEBC showed a dose-dependent impairment (Steinmetz and Freeman 2010). However, administration of WIN55,212-2 produced a larger impairment than that of SR141716A. Similarly, human and CB1R knockout mouse studies have shown deficits in acquisition of dEBC (Kishimoto and Kano 2006; Skosnik et al. 2008). Humans, who were currently using cannabis but not intoxicated at the time of the experiment, exhibited marked decrements in acquisition of dEBC compared to controls (Skosnik et al. 2008). CB1R knockout mice were also unable to acquire dEBC at a normal rate (Kishimoto and Kano 2006). Thus, manipulations of CB1R function impair acquisition of $\mathrm{AEBC}$ in mice, rats, and humans.

The role of CB1Rs after learning has already occurred (retention) has not been studied. It was previously hypothesized that CB1Rs play a role in induction of synaptic plasticity within the cerebellar cortex (Steinmetz and Freeman 2010), suggesting that manipulating these receptors should not affect retention but will impair extinction, a type of inhibitory learning. Both the CB1R knockout mouse and human studies examined extinction and found no significant differences. A limitation of these studies is that similar levels of conditioning were not established prior to extinction training. Skosnik et al. (2008) administered extinction after 10 blocks of acquisition in humans; a decline in conditioned response (CR) percentage was observed in both the cannabis and control groups. However, prior to extinction, performance of the

\footnotetext{
${ }^{1}$ Corresponding author.
}

E-mail john-freeman@uiowa.edu.

Article is online at http://www.learnmem.org/cgi/doi/10.1101/Im.2254111. control group was $\sim 80 \%$ CRs and the cannabis group was $\sim 50 \%$ CRs (Skosnik et al. 2008). Kishimoto and Kano (2006) also gave extinction to CB1R knockout mice and mice administered SR141716A and found no deficit in either group. Similar to the human study, equal levels of CR percentage were not established prior to extinction for the CB1R knockout mice; the CB1R knockout mice exhibited $\sim 30 \%$ CRs compared to $\sim 75 \%$ for controls. However, the mice administered SR141716A during extinction had similar levels of learning prior to the start of extinction. Thus, the absence of significant effects in human users and CB1R knockout mice during extinction could be due to differences prior to extinction and not to the effects of CB1R manipulations. It is important to examine the effects of CB1R agonists/ antagonists on extinction because alterations in synaptic transmission in the cerebellar cortex are hypothesized to impair synaptic plasticity underlying behavioral inhibition and consequently impair extinction.

The current study used the CB1R agonist WIN55,212-2 and antagonist SR141716A to examine the role of the CB1R in retention and extinction of dEBC. All rats were trained for five CS-US sessions to reach asymptotic levels of learning. Rats were given subcutaneous administration of $3 \mathrm{mg} / \mathrm{kg}$ of WIN55,212-2 or $5 \mathrm{mg} / \mathrm{kg}$ of SR141716A on the sixth CS-US session, followed by a session with vehicle administration. They were then administered WIN55,212-2, SR141716A, or vehicle during two sessions of CS-alone extinction. The doses of the agonist and antagonist were chosen because both produced impairments in acquisition of dEBC in a previous study (Steinmetz and Freeman 2010). The $3 \mathrm{mg} / \mathrm{kg}$ dose of WIN55,212-2 produced significant impairments compared to smaller dose of WIN55,212-2 (1 and $2 \mathrm{mg} / \mathrm{kg}$ ). For SR141716A, it was reported that $5 \mathrm{mg} / \mathrm{kg}$ produced maximal effects and did not differ from a lower dose of SR141716A $(3 \mathrm{mg} / \mathrm{kg})$ (Steinmetz and Freeman 2010).

\section{Results}

\section{Experiment 1: WIN55,212-2}

\section{Retention}

Rats were trained for $5 \mathrm{~d}$ prior to subcutaneous injections of the CB1R agonist WIN55,212-2 (3 mg/ $\mathrm{kg})$ on the sixth day of CS-US 
training. On the day following WIN55,212-2 injections, rats received an injection of the vehicle. In order to confirm that rats learned prior to the retention session, a repeated measures analysis of variance (ANOVA) was completed for the first five sessions and revealed a main effect of Session for CR percentage (Fig. 1, P1-5), $F_{(6,10)}=18.764, P<0.001$; CR amplitude, $F_{(6,10)}=8.924$, $P=0.002$; CR onset latency, $F_{(6,10)}=7.729, P<0.001$; and $\mathrm{CR}$ peak latency, $F_{(6,10)}=5.618, P=0.009$. The session effects indicate an increase in CR percentage, amplitude, and also changes in CR timing. Taken together, these results indicate that rats learned dEBC prior to the retention test.

A paired samples $t$-test was completed to examine effects of WIN55,212-2 on Session 6 compared to vehicle administration on Session 7. Administration of WIN55,212-2 (Fig. 1, WIN) caused a small deficit in CR percentage $(75.58 \pm 3.78)$ as compared to vehicle $(89.40 \pm 1.99)$, paired $t_{(15)}=4.08, P<0.001$. To further examine this effect each session was separated into 10 blocks (Fig. 2). A 2 (Session) $\times 10$ (Block) repeated measures ANOVA revealed a significant Session $\times$ Block interaction, $F_{(1,8)}=2.017$, $P=0.042$. Post-hoc tests (Tukey's Honestly Significant Difference) revealed significantly lower CR percentages for blocks $1-3,8$, and $10(P<0.05)$ during the session in which WIN55,212-2 was administered, compared to vehicle. A $t$-test was completed for CR amplitude and found a significant difference between the WIN55,212-2 and vehicle administration sessions, paired $t_{(15)}=$ $3.82, P=0.002$. Paired samples $t$-tests on the CR onset and peak latency data indicated that WIN55,212-2 administration had no significant effect as compared to vehicle administration. Repeated measures ANOVAs for block data could not be completed for CR onset latency, CR peak latency, or CR amplitudes due to blocks without CRs on the test trial. UR amplitudes were compared during CS-US retention sessions in order to examine possible effects of WIN55,212-2 on performance, but no significant difference was found.

\section{Extinction}

After completing the $7 \mathrm{~d}$ of paired CS-US training, rats were given two sessions of extinction training, each consisting of 100 CS-alone trials. Rats were randomly assigned to receive administration of either WIN55,212-2 or vehicle prior to the extinction

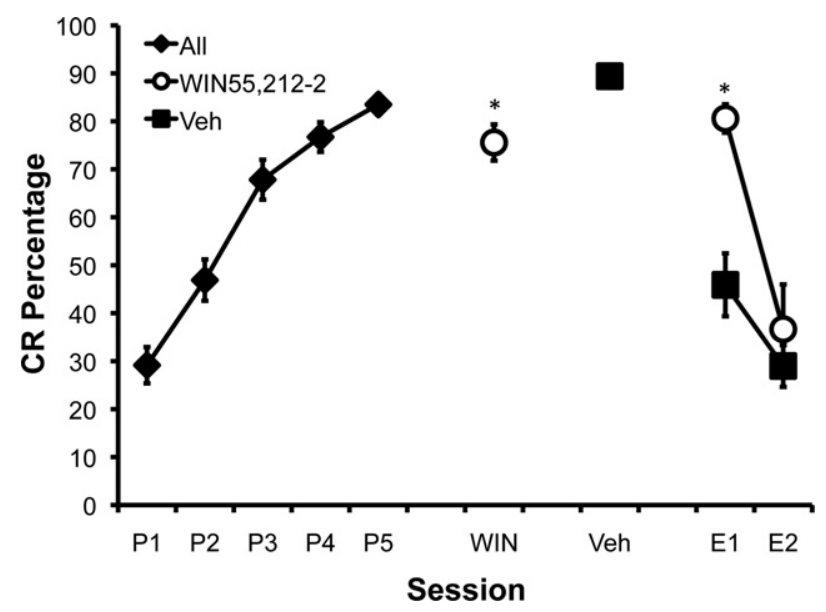

Figure 1. Mean $\pm S E$ conditioned response percentage prior to injections (P1-P5), during WIN55,212-2 administration (WIN) and during vehicle administration (Veh). Rats were given either WIN55,212-2 (open circles) or vehicle (filled squares) during two sessions of CS-alone extinction (E1 and E2). $\left({ }^{*}\right)$ Asterisks indicate significant group differences $(P<$ 0.05).

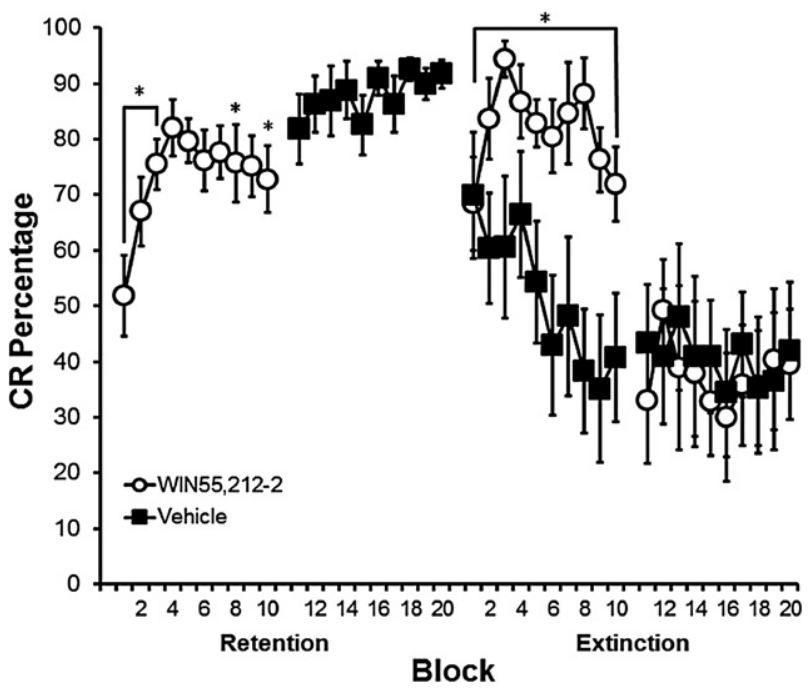

Figure 2. Mean \pm SE conditioned response percentage during blocks of retention and extinction. Rats were administered WIN55,212-2 (open circles) or vehicle (filled squares) during two sessions of CS-alone extinction. $\left.{ }^{*}\right)$ Asterisks indicate significant group differences $(P<0.05)$.

sessions. In order to examine potential differences prior to extinction, a one-way ANOVA for CR percentage was completed for the CS-US session prior to extinction and found no significant differences between groups that were to receive WIN55,212-2 or vehicle during extinction. CR percentage data during extinction were examined using a repeated measures ANOVA (Fig. 1, E1-2). The ANOVA revealed a significant Session $\times$ Group interaction for CR percentage, $F_{(1,14)}=8.407, P=0.012$. Post-hoc tests revealed a higher CR percentage in the group given WIN55,212-2 relative to the control group during the first session of extinction $(P<$ $0.001)$ but not during the second session. To further examine extinction differences, a 2 (Group) $\times 2$ (Session) $\times 10$ (Block) ANOVA was computed for block data during each session for CR percentage. A Group $\times$ Session interaction, $F_{(1,14)}=13.000, P=$ 0.003 , was found which was caused by a higher percentage of CRs in the groups given WIN55,212-2 during the first session of extinction but not the second session. Repeated measures ANOVAs were conducted for CR onset latency, peak latency, and amplitude data (Fig. 2) and revealed no significant group differences. Similar to the retention analysis, block analysis for CR onset latency, CR peak latency, and CR amplitudes could not be conducted due to the small number of CRs on CS-alone trials.

\section{Experiment 2: SR141716A}

\section{Retention}

After $5 \mathrm{~d}$ of CS-US training, rats were given a subcutaneous injection of the CB1R antagonist SR141716A $(5 \mathrm{mg} / \mathrm{kg})$ followed the next day by administration of the vehicle. Repeated measures ANOVAs were completed for CR percentage, CR amplitude, CR onset latency, and CR peak latency for Sessions 1-5 to confirm that learning occurred prior to the retention test. The ANOVAs revealed main effects of Session over the $5 \mathrm{~d}$ of paired training for CR percentage (Fig. 3), $F_{(6,10)}=20.197, P<0.001$; onset latency, $F_{(6,10)}=7.729, P<0.001$; peak latency, $F_{(6,10)}=5.618$, $P=0.009 ;$ and amplitude, $F_{(6,10)}=11.564, P<0.001$. Taken together, these data indicate that the rats learned prior to the retention test.

Injections of SR141716A on Session 6 did not cause a deficit in CR percentage, amplitude, onset latency, or peak latency 


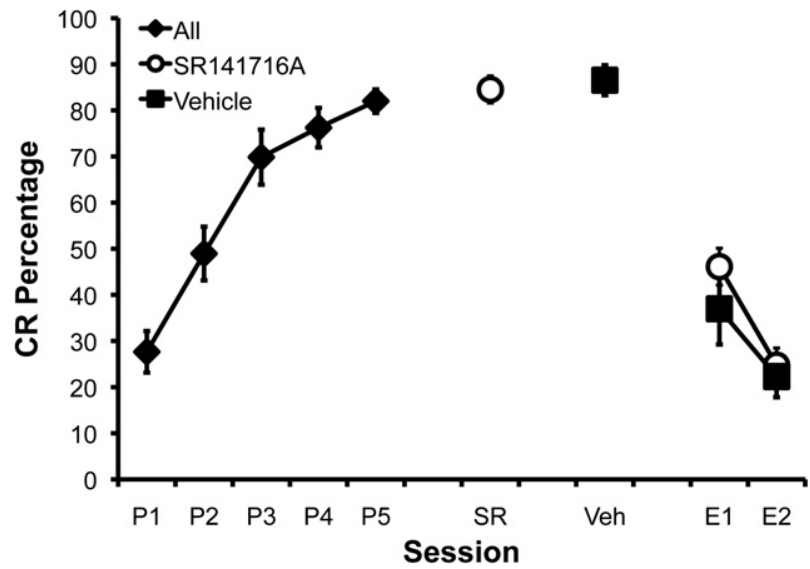

Figure 3. Mean $\pm \mathrm{SE}$ conditioned response percentage prior to injection (P1-P5), during SR141716A (SR) and during vehicle administration (Veh). Rats were given either SR141716A (open circles) or vehicle (filled squares) during two sessions of CS-alone extinction (E1 and E2).

compared to vehicle administration, as revealed by a paired samples $t$-test (Fig. 3). Each session was then subdivided into 10 blocks (Fig. 4), and an ANOVA revealed no significant effects of Session or a Session $\times$ Block interaction for the CR percentage data. The amplitude of the UR between the SR141716A and vehicle administration sessions was compared to examine potential drug effects on performance, and no significant difference was revealed.

\section{Extinction}

As in Experiment 1, rats were randomly assigned to receive administration of either SR141716A or vehicle during two CS-alone extinction sessions. In order to examine differences prior to extinction, a one-way ANOVA was completed for the session prior to extinction and found no significant differences between the groups that received SR141716A or vehicle. The effects of SR141716A on CR percentage were compared to vehicle administration during extinction using a repeated measures ANOVA (Fig. 3, E1-2). The ANOVA revealed a main effect of Session for CR percentage, $F_{(1,14)}=24.767, P<0.001$, but no main effect of Group. A 2 (Group) $\times 2$ (Session) $\times 10$ (Block) repeated measures ANOVA was completed for the rats administered SR141716A compared to control rats (Fig. 4). The ANOVA revealed a significant interaction for Session $\times$ Block, $F_{(1,9)}=3.771, P=0.007$, but no effects involving the Group factor. Repeated measures ANOVAs were also completed for CR onset latency, peak latency, and amplitude, which revealed no significant group differences. Taken together these findings indicate that SR141716A did not affect extinction of dEBC.

\section{Discussion}

In the current study, rats were given five CS-US sessions prior to manipulating CB1Rs during a CS-US retention test. Subcutaneous injections of the CB1R agonist WIN55,212-2 mildly impaired retention, whereas injections of the CB1R antagonist SR141716A had no effect. During extinction, rats given injections of WIN55,212-2 exhibited a resistance to extinction, whereas SR141716A injections did not affect extinction. Prior to the extinction phase, all rats exhibited a similar level of conditioning, suggesting that differences during extinction were due to manipulations of CB1R and not to pre-existing learning differences.
Previous studies using manipulations of CB1Rs in mammals demonstrated impaired acquisition of dEBC (Kishimoto and Kano 2006; Skosnik et al. 2008; Steinmetz and Freeman 2010). Acquisition of $\mathrm{dEBC}$ is dependent upon the cerebellar cortex and anterior interpositus nucleus (Thompson and Steinmetz 2009). The synaptic mechanism in the cerebellar cortex underlying dEBC is long-term depression (LTD) of parallel fiber-toPurkinje cell synapses (Ito and Kano 1982; Raymond et al. 1996; Mauk and Donegan 1997). Cerebellar LTD results in pauses in Purkinje cell simple spikes that temporarily release the interpositus nucleus from inhibition (Pugh and Raman 2009), which has been hypothesized to promote induction of mossy fiber to interpositus nucleus long-term potentiation (Mauk and Donegan 1997; Medina et al. 2000). Learning-related pauses in Purkinje cell firing have been reported in studies examining extracellular activity during dEBC (Hesslow and Ivarsson 1994; Green and Steinmetz 2005; Jirenhed et al. 2007; Jirenhed and Hesslow 2011). Moreover, CB1R knockout mice and mice injected with SR14716A do not exhibit LTD (Safo and Regehr 2005), and manipulations of CB1R function, using either WIN55,212-2 or SR141716A, have been shown to impair induction of parallel fiber-Purkinje cell LTD in vitro (Lévénés et al. 1998; Safo and Regehr 2005) and impair acquisition of dEBC (Steinmetz and Freeman 2010). In the current study, WIN55,212-2 was shown to slightly impair performance of conditioned responses after learning. WIN55,212-2 decreases glutamate release at parallel fiber-Purkinje cell synapses, which may partially reverse previously formed LTD. In contrast, increasing glutamate release with SR141716A appears to have no impact on already formed LTD in vivo. Deficits in LTD with WIN55,212-2 could also be related to the decrease in GABA release from basket and stellate cells. Another possibility is that the minor deficit in CR percentage during retention testing with WIN55,212-2 was caused by reduced glutamate release in the interpositus nucleus. Lastly, consideration should be given to the possibility of a state-dependent effect during retention. The group given WIN55,212-2 showed an initial drop in CRs followed by an increase within the retention session, which could be partial relearning in the drug state. Overall, results from this study suggest that decreasing glutamate in the



Figure 4. Mean $\pm \mathrm{SE}$ conditioned response percentage during blocks of retention and extinction. Rats were administered SR141716A (open circles) or vehicle (filled squares) during two sessions of CS-alone extinction. 
cerebellum mildly disrupts already formed learning, whereas increasing glutamate release has little effect.

Few studies have examined the neural substrates of extinction of dEBC (Medina et al. 2002; Robleto et al. 2004). A computer simulation of the cerebellum suggests that the LTD that forms during acquisition is reversed, becoming LTP during CS-alone extinction (Medina et al. 2002; Mauk and Ohyama 2004). The signal for inducing LTP and thus extinction is cerebellar inhibition of the inferior olive in the absence of the US during CS-alone trials (Medina et al. 2002). Blockade of cerebellar inhibition of the inferior olive impairs extinction by maintaining climbing fiber activity and cortical LTD (Medina et al. 2002). Reversal of LTD-like decreases in simple spike activity back to baseline has been reported experimentally (Jirenhed et al. 2007). Moreover, lesions of the anterior lobe of the cerebellar cortex have been shown to impair extinction of dEBC (Perrett and Mauk 1995). The current study found that WIN55,212-2 impaired extinction of the CR during Session 1 but not during Session 2. The presynaptic action of WIN55, 212-2 may impair induction of a short-term form of parallel fiber-to-Purkinje cell LTP but not long-term LTP (Hirano 1990; Salin et al. 1996; Lev-Ram et al. 2002, 2003; Jörntell and Hansel 2006). As suggested by Ohyama et al. (2010), presynaptic LTP may be relatively short lived, whereas post-synaptic LTP might maintain extinction. Thus, WIN55-5,212-2 may have selectively impaired presynaptic LTP without affecting post-synaptic LTP mechanisms, resulting in an absence of extinction during Session 1 but normal extinction during Session 2.

Areas outside of the cerebellar cortex that play a role in $\mathrm{dEBC}$, including the auditory system, red nucleus, interpositus nucleus, and hippocampus, also contain CB1Rs (Herkenham et al. 1991; Suárez et al. 2008). However, studies with human cannabis users and CB1R knockout mice have shown unimpaired trace EBC, which requires hippocampus, auditory system, cerebellar deep nuclei, and red nucleus (Kishimoto and Kano 2006; Edwards and Skosnik 2007). Thus, the deficit in retention and extinction may be caused by the action of WIN55,212-2 on cerebellar cortical function. However, this issue can only be resolved by using localized infusions of WIN55,212-2 and SR141716A into the cerebellar cortex.

In summary, the current study is the first to demonstrate deficits in retention and extinction of dEBC with manipulations of the CB1Rs. WIN55,212-2, but not SR141716A, slightly impairs retention of $\mathrm{dEBC}$. Previous work in both human and knockout mice found no impairments in extinction of dEBC (Kishimoto and Kano 2006; Skosnik et al. 2008). However, learning was not matched prior to extinction in these studies. The current study controlled for pre-extinction conditioning, with all rats at similar levels of learning prior to extinction. Administration of WIN55,212-2 impaired extinction of dEBC, whereas SR141716A had no effect. The WIN55,212-2-induced extinction deficit may be caused by impaired LTP induction at parallel fiber synapses with Purkinje cells. Additional studies are needed to elucidate the cellular mechanisms underlying the retention and extinction deficits.

\section{Materials and Methods}

\section{Subjects}

Subjects were 32 male Long-Evans rats, 250-350 g at the beginning of the experiment. The rats were housed in the Spence Laboratories of Psychology at the University of Iowa with a 12-h light-dark cycle and were given ad libitum access to food and water.

\section{Surgery}

One week prior to training, rats were removed from their home cage and anesthetized with isoflurane. After the onset of anesthesia, the rats were fitted with differential electromyograph (EMG) electrodes (stainless steel) implanted into the upper left orbicularis oculi muscle. The ground electrode was a silver wire attached to a stainless steel skull screw. The EMG electrode leads terminated in gold pins in a plastic connector. A bipolar stimulating electrode (Plastics One) for delivering the shock US was implanted subdermally, caudal to the left eye.

\section{Apparatus}

The conditioning apparatus consisted of four small-animal sound attenuation chambers (BRS/LVE). Within each sound attenuation chamber was a small animal operant chamber (BRS/LVE) where the rats were located during conditioning. One wall of the operant chamber was fitted with two speakers from which the CS was presented. The electrode leads from the rat's headstage were connected to peripheral equipment. Computer software controlled the delivery of stimuli and the recording of eyelid EMG activity (JSA Designs). The shock US (2-3.5 mA, DC constant current) was delivered through a stimulus isolator (model number 365A, World Precision Instruments). EMG activity was recorded differentially, filtered $(500-5000 \mathrm{~Hz})$, and integrated as described in other reports (Nicholson and Freeman 2002; Freeman et al. 2005).

\section{Conditioning procedures}

Rats recovered from surgery for 1 wk prior to the initiation of training. All rats completed nine consecutive daily sessions of training. Sessions 1-7 consisted of 10 blocks of nine paired CS-US presentations and 1 CS-alone probe trial. Prior to Session 6, rats were administered either WIN55,212-2 or SR141716A. They were given vehicle prior to Session 7. Sessions 8 and 9 were the extinction phase of the experiment in which $100 \mathrm{CS}$-alone trials were presented. Rats were given WIN55,212-2 $(n=8)$, SR141716A $(n=8)$, or vehicle $(n=16)$ for both sessions of extinction. The CS was a $400-\mathrm{msec}$ tone $(2 \mathrm{kHz} ; 85 \mathrm{~dB})$. The CS terminated with a 25-msec shock US. The shock intensity was adjusted for each rat to elicit a blink and slight head movement (range $=2-3.5 \mathrm{~mA}$ ). CRs were defined as EMG activity that exceeded a threshold of 0.4 unit (amplified and integrated units) above the baseline mean during the CS period after 80 msec. EMG responses that exceeded the threshold during the first $80 \mathrm{msec}$ of the CS period were defined as startle responses. On CS-alone probe trials, the duration for scoring CRs was extended beyond the CS to the end of the trial period $(1.0 \mathrm{sec})$. CR amplitude and latency measures (CR Onset and Peak) were taken from CS-alone trials with CRs. URs were defined as responses that crossed the threshold after the offset of the US to avoid the stimulation artifact.

\section{Pharmacological activation/blockade of $\mathrm{CB1}$ receptors}

The CB1 agonist WIN55,212-2[(R)-(+)-[2,3-Dihydro-5-methyl3-(4-morpholinylmethyl)pyrrolo[1,2,3-de)-1,4-benzoxazin6-yl]-1-napthalenylmethanone] and antagonist SR141716A [N-piperidino-5-(4-chlorophenyl)-1-(2,4-dichlorophenyl)-4methyl-3-pyrazole carboxamide] were administered subcutaneously $30 \mathrm{~min}$ before each daily training session. WIN55,212-2 binds with high affinity to CB1R and weakly to CB2R, whereas SR141716A binds exclusively to CB1R (Pertwee 1997; Howlett et al. 2002). WIN55,212-2 was administered at a dose of $3 \mathrm{mg} /$ $\mathrm{kg}$, and SR141716A was administered at a dose of $5 \mathrm{mg} / \mathrm{kg}$. WIN55,212-2 was dissolved in a vehicle of 1:1:18 solution of ethanol, cremaphor, saline, and SR141716A was dissolved in a vehicle of 1:1:18 solution of ethanol, tween80, saline. WIN55,212-2 was purchased from Sigma/RBI, and SR141716A was a generous gift from NIDA Drug Supply Program. In a previous study, rats treated with WIN55,212-2 at $3 \mathrm{mg} / \mathrm{kg}$ showed marked deficits compared to lower doses during dEBC. SR141716A at $5 \mathrm{mg} / \mathrm{kg}$ also impaired acquisition of dEBC, but this dose did not differ from the $3 \mathrm{mg} / \mathrm{kg}$ dose, indicating that a higher concentration would not be more effective (Steinmetz and Freeman 2010). 


\section{Acknowledgments}

We thank Johanna Levine and Sarah Galvin for data collection throughout the project. This work was supported by NIMH grant MH080005 to J.H.F.

\section{References}

Attwell PJ, Rahman S, Yeo CH. 2001. Acquisition of eyeblink conditioning is critically dependent on normal function in cerebellar cortical lobule HVI. J Neurosci 21: 5715-5722.

Chen L, Bao S, Lockard JM, Kim JK, Thompson RF. 1996. Impaired classical eyeblink conditioning in cerebellar-lesioned and Purkinje cell-degeneration (pcd) mutant mice. J Neurosci 16: 2829-2838.

Chen L, Bao S, Thompson RF. 1999. Bilateral lesions of the interpositus nucleus completely prevent eyeblink conditioning in Purkinje cell-degeneration mutant mice. Behav Neurosci 113: 204-210.

Edwards CR, Skosnik PD. 2007. Cerebellar-dependent learning as a neurobehavioral index of the cannabinoid system. Crit Rev Neurobiol 19: $29-57$.

Freeman JH, Halverson HE, Poremba A. 2005. Differential effects of cerebellar inactivation on eyeblink conditioned excitation and inhibition. J Neurosci 25: 889-895.

Garcia KS, Steele PM, Mauk MD. 1999. Cerebellar cortex lesions prevent acquisition of conditioned eyelid responses. J Neurosci 19: 10940-10947.

Green JT, Steinmetz JE. 2005. Purkinje cell activity in the cerebellar anterior lobe after rabbit eyeblink conditioning. Learn Mem 12: 260-269.

Herkenham M, Lynn AB, Little MD, Johnson MR, Melvin LS, de Costa BR, Rice KC. 1990. Cannabinoid receptor localization in brain. Proc Natl Acad Sci 87: 1932-1936.

Herkenham M, Lynn AB, Johnson MR, Melvin LS, de Costa BR, Rice KC. 1991. Characterization and localization of cannabinoid receptors in rat brain: A quantitative in vitro autoradiographic study. J Neurosci 11: 563-583.

Hesslow G, Ivarsson M. 1994. Suppression of cerebellar Purkinje cells during conditioned responses in ferrets. Neuroreport 5: 649-652.

Hirano T. 1990. Depression and potentiation of the synaptic transmission between a granule cell and a Purkinje cell in rat cerebellar culture. Neurosci Lett 119: 141-144.

Howlett AC, Barth F, Bonner TI, Cabral G, Casellas P, Devane WA Felder CC, Herkenham M, Mackie K, Martin BR, et al. 2002. International Union of Pharmacology. XXVII. Classification of cannabinoid receptors. Pharmacol Rev 54: 161-202.

Ito M, Kano M. 1982. Long-lasting depression of parallel fiber-Purkinje cell transmission induced by conjunctive stimulation of parallel fibers and climbing fibers in the cerebellar cortex. Neurosci Lett 33: 253-258.

Jirenhed DA, Hesslow G. 2011. Learning stimulus intervals-adaptive timing of conditioned Purkinje cell responses. Cerebellum. doi: 10.1007/ s12311-011-0264-3.

Jirenhed DA, Bengtsson F, Hesslow G. 2007. Acquisition, extinction, and reacquisition of a cerebellar cortical memory trace. J Neurosci 27: 2493-2502.

Jörntell H, Hansel C. 2006. Synaptic memories upside down: Bidirectional plasticity at cerebellar parallel fiber-Purkinje cell synapses. Neuron 52: 227-238.

Kishimoto Y, Kano M. 2006. Endogenous cannabinoid signaling through the CB1 receptor is essential for cerebellum-dependent discrete motor learning. J Neurosci 26: 8829-8837.

Lavond DG, Steinmetz JE. 1989. Acquisition of classical conditioning without cerebellar cortex. Behav Brain Res 33: 113-164.
Lévénés C, Daniel H, Soubrié P, Crépel F. 1998. Cannabinoids decrease excitatory synaptic transmission and impair long-term depression in rat cerebellar Purkinje cells. J Physiol 510: $867-879$.

Lev-Ram V, Wong ST, Storm DR, Tsien RY. 2002. A new form of cerebellar long-term potentiation is postsynaptic and depends on nitric oxide but not cAMP. Proc Natl Acad Sci 99: 8389-8393.

Lev-Ram V, Mehta SB, Kleinfeld D, Tsien RY. 2003. Reversing cerebellar long-term depression. Proc Natl Acad Sci 100: 15989-15993.

Mauk MD, Donegan NH. 1997. A model of Pavlovian eyelid conditioning based on the synaptic organization of the cerebellum. Learn Mem 4: $130-158$.

Mauk MD, Ohyama T. 2004. Extinction as new learning versus unlearning: Considerations from a computer simulation of the cerebellum. Learn Mem 11: 566-571.

Medina JF, Nores WL, Ohyama T, Mauk MD. 2000. Mechanisms of cerebellar learning suggested by eyelid conditioning. Curr Opin Neurobiol 10: 717-724.

Medina JF, Nores WL, Mauk MD. 2002. Inhibition of climbing fibers is a signal for the extinction of conditioned eyelid responses. Nature 416: 330-333.

Nicholson DA, Freeman JH. 2002. Medial dorsal thalamic lesions impair blocking and latent inhibition of the conditioned eyeblink response in rats. Behav Neurosci 116: 276-285.

Nolan BC, Freeman JH. 2006. Purkinje cell loss by OX7-saporin impairs acquisition and extinction of eyeblink conditioning. Learn Mem 13: 359-365.

Ohyama T, Voicu H, Kalmbach B, Mauk MD. 2010. A decrementing form of plasticity apparent in cerebellar learning. J Neurosci 30: 16993-17003.

Ong WY, Mackie K. 1999. A light and electron microscopic study of the CB1 cannabinoid receptor in primate brain. Neuroscience 92: $1177-1191$.

Perrett SP, Mauk MD. 1995. Extinction of conditioned eyelid responses requires the anterior lobe of cerebellar cortex. J Neurosci 15: 2074-2080.

Pertwee RG. 1997. Pharmacology of cannabinoid CB1 and CB2 receptors. Pharmacol Ther 74: 129-180.

Pugh JR, Raman IM. 2009. Nothing can be coincidence: Synaptic inhibition and plasticity in the cerebellar nuclei. Trends Neurosci 32: $170-177$.

Raymond JL, Lisberger SG, Mauk MD. 1996. The cerebellum: A neuronal learning machine? Science 272: 1126-1131.

Robleto K, Poulos AM, Thompson RF. 2004. Brain mechanisms of extinction of the classically conditioned eyeblink response. Learn Mem 11: $517-524$

Safo PK, Regehr WG. 2005. Endocannabinoids control the induction of cerebellar LTD. Neuron 48: 647-659.

Salin PA, Malenka RC, Nicoll RA. 1996. Cyclic AMP mediates a presynaptic form of LTP at cerebellar parallel fiber synapses. Neuron 16: 797-803.

Skosnik PD, Edwards CR, O’Donnell BF, Steffen A, Steinmetz JE, Hetrick WP. 2008. Cannabis use disrupts eyeblink conditioning: Evidence for cannabinoid modulation of cerebellar-dependent learning. Neuropsychopharmacology 33: 1432-1440.

Steinmetz AB, Freeman JH. 2010. Central cannabinoid receptors modulate acquisition of eyeblink conditioning. Learn Mem 17: 571-576.

Suárez J, Bermúdez-Silva FJ, Mackie K, Ledent C, Zimmer A, Cravatt BF, de Fonseca FR. 2008. Immunohistochemical description of the endogenous cannabinoid system in the rat cerebellum and functionally related nuclei. J Comp Neurol 509: 400-421.

Thompson RF, Steinmetz JE. 2009. The role of the cerebellum in classical conditioning of discrete behavioral responses. Neuroscience 162: $732-755$.

Received April 26, 2011; accepted in revised form August 10, 2011. 


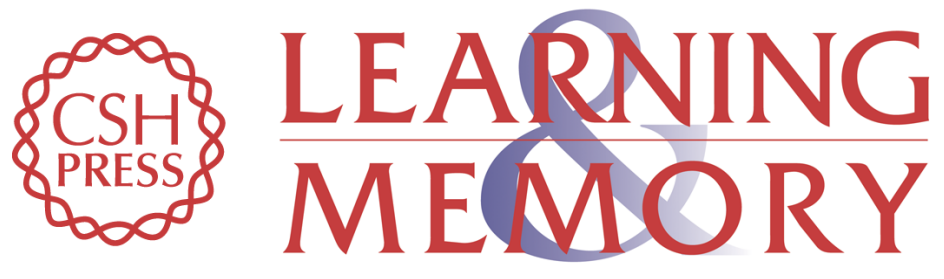

\section{Retention and extinction of delay eyeblink conditioning are modulated by central cannabinoids}

Adam B. Steinmetz and John H. Freeman

Learn. Mem. 2011, 18:

Access the most recent version at doi:10.1101//m.2254111

References This article cites 40 articles, 20 of which can be accessed free at: http://learnmem.cshlp.org/content/18/10/634.full.html\#ref-list-1

License

Email Alerting Receive free email alerts when new articles cite this article - sign up in the box at the Service top right corner of the article or click here. 